\title{
PARTICIPATION IN MULTILATERAL TREATIES
}

\section{KO SWAN SIK*}

\section{Editorial introduction}

In emphasizing the actual practice of Asian States in the field of international law it has been considered useful to record their participation in open, multilateral law-making treaties which by their nature aim at world-wide adherence. The determination of the priority in which treaties qualify for inclusion is admittedly subjectively prescribed. In view of the limited space available a choice has been made among the great number of treaties included in the sources of information, which were at the Editors' disposal, preparing the manuscript of the present volume. As to the countries included in the survey it has been considered preferable not to include for the time being the Arab countries, Turkey, and Cyprus, despite the fact that these countries are often considered to belong to Asia, either in general or for specific purposes. Similarly, it has been decided not to include the Pacific States east of Papua New Guinea and the Philippines.

It is the Editors' intention to include tables on the status of other treaties in following volumes of the Yearbook, and to record new developments with respect to treaties on which a full table has been published previously. After the publication of a full cycle of tables of chosen treaties a new updated series may be contemplated.

Note:

- Data are mainly derived from Multilateral Treaties deposited with the Secretary General Status as at 31 December 1990 (ST/LEG/SER.9). Additional sources are mentioned separately at the relevant place.

- No indication is given of reservations and declarations made.

- Sig. = signature; Cons. $=$ consent to be bound.

\section{LAW OF TREATIES}

\section{Convention on the Law of Treaties \\ Vienna, 1969}

Entry into force: 27 Jan. 1980

\begin{tabular}{lll|lll} 
State & Sig. & Cons. & State & Sig. & Cons. \\
Afghanistan & 23 May 69 & & Mongolia & & 16 May 88 \\
$\begin{array}{l}\text { Cambodia } \\
\text { Iran }\end{array}$ & 23 May 69 & & Nepal & 23 May 69 & \\
Japan & 23 May 69 & & Pakistan & 29 Apr 70 & \\
Korea (Rep) & 27 Nov 69 & 27 Jul 81 & Philippines & 23 May 69 & 15 Nov 72
\end{tabular}

* General Editor

Ko Swan Sik et al. (eds.), Asian Yearbook of International Law, 183-196.

(c) 1993 Kluwer Academic Publishers. Printed in the Netherlands. 


\section{LAW OF THE SEA}

\section{United Nations Convention on the Law of the Sea}

Montego Bay, Jamaica, 1982

Entry into force: -

(1991 data courtesy of Netherlands Ministry of Foreign Affairs)

\begin{tabular}{|c|c|c|c|c|c|}
\hline State & Sig. & Cons. & State & Sig. & Cons. \\
\hline Afghanistan & $18 \operatorname{Mar} 83$ & & Malaysia & 10 Dec 82 & \\
\hline Bangladesh & 10 Dec 82 & & Maldives & 10 Dec 82 & \\
\hline Bhutan & 10 Dec 82 & & Mongolia & 10 Dec 82 & \\
\hline Brunei & 5 Dec 84 & & Myanmar & 10 Dec 82 & \\
\hline Cambodia & 1 Jul 83 & & Nepal & 10 Dec 82 & \\
\hline China & $10 \mathrm{Dec} 82$ & & Pakistan & 10 Dec 82 & \\
\hline India & 10 Dec 82 & & Papua New Guinea & 10 Dec 82 & \\
\hline Indonesia & $10 \operatorname{Dec} 82$ & 3 Feb 86 & Philippines & $10 \operatorname{Dec} 82$ & 8 May 84 \\
\hline Iran & 10 Dec 82 & & Seychelles & 10 Dec 82 & 16 Sep 91 \\
\hline Japan & 7 Feb 83 & & Singapore & 10 Dec 82 & \\
\hline Korea (DPR) & $10 \mathrm{Dec} 82$ & & Sri Lanka & $10 \operatorname{Dec} 82$ & \\
\hline Korea (Rep) & $14 \mathrm{Mar} 83$ & & Thailand & 10 Dec 82 & \\
\hline Laos & 10 Dec 82 & & Vietnam & 10 Dec 82 & \\
\hline
\end{tabular}

INTERNATIONAL TRADE LAW AND COMMERCIAL ARBITRATION

(Data also from A/CN.9/353 - 5 Jun. 1991)

Convention on the Recognition and Enforcement of Foreign Arbitral Awards New York, 1958

Entry into force: 7 Jun. 1959

\begin{tabular}{llr|llc} 
State & Sig. & Cons. & State & Sig. & Cons. \\
Cambodia & & 5 Jan 60 & Malaysia & & 5 Nov 85 \\
China & & 22 Jan 87 & Pakistan & 30 Dec 58 & \\
India & 13 Jul 60 & Philippines & 10 Jun 58 & 6 Jul 67 \\
Indonesia & 10 Jun 58 & Singapore & & 21 Aug 86 \\
Japan & & 20 Jun 61 & Sri Lanka & 30 Dec 58 & 9 Apr 62 \\
Korea (Rep.) & & 8 Feb 73 & Thailand & & 21 Dec 59
\end{tabular}

\section{Convention on the Limitation Period in the International Sale of Goods}

New York, 1974

Entry into force: 1 Aug. 1988

State

Sig. Cons.

Mongolia

14 Jun 74 
United Nations Convention on the Carriage of Goods by Sea

Hamburg, 1978

Entry into force: -

\begin{tabular}{lll|lll} 
State & Sig. & Cons. & State & Sig. & Cons. \\
Pakistan & $8 \mathrm{Mar} 79$ & & Singapore & $31 \mathrm{Mar} 78$ & \\
Philippines & 14 Jun 78 & & & &
\end{tabular}

\section{United Nations Convention on Contracts for the International Sale of Goods Vienna, 1980}

Entry into force: 1 Jan. 1988

\begin{tabular}{lll|lll} 
State & Sig. & Cons. & State & Sig. & Cons. \\
China & 30 Sep 81 & 11 Dec 86 & Singapore & 11 Apr 80 &
\end{tabular}

United Nations Convention on the Liability of Operators of Transport Terminals in International Trade

Vienna, 1991

Entry into force: -

State

Sig. Cons.

Philippines $\quad 19$ Apr 91

\section{PROTECTION OF THE ENVIRONMENT}

Convention for the Protection of the Ozone Layer

Vienna, 1985

Entry into force: 22 Sep. 1988

(Status as at 31 Jan. 1992, from UNEP)

\begin{tabular}{|c|c|c|c|c|c|}
\hline State & Sig. & Cons. & State & Sig. & Cons. \\
\hline Bangladesh & & 2 Aug 90 & Malaysia & & 29 Aug 89 \\
\hline Brunei & & 26 Jul 90 & Maldives & & 26 Apr 88 \\
\hline China & & $11 \operatorname{Sep} 89$ & Philippines & & 17 Jul 91 \\
\hline India & & $18 \operatorname{Mar} 91$ & Singapore & & 5 Jan 89 \\
\hline Iran & & 3 Oct 90 & Sri Lanka & & $15 \operatorname{Dec} 89$ \\
\hline Japan & & 30 Sep 88 & Thailand & & 7 Jul 89 \\
\hline
\end{tabular}




\section{Protocol on Substances that Deplete the Ozone Layer \\ Montreal, 1987 \\ Entry into force: $1 \mathrm{Jan} .1989$ \\ (Status as at 31 Jan. 1992, from UNEP)}

\begin{tabular}{|c|c|c|c|c|c|}
\hline State & Sig. & Cons. & State & Sig. & Cons. \\
\hline Bangladesh & & 2 Aug 90 & Maldives & 12 Jul 88 & 16 May 89 \\
\hline China & & 14 Jun 91 & Philippines & 14 Sep 88 & \\
\hline Indonesia & $21 \mathrm{JuI} 88$ & & Singapore & & $5 \operatorname{Jan} 89$ \\
\hline Iran & & 3 Oct 90 & Sri Lanka & & 15 Dec 89 \\
\hline Japan & 16 Sep 87 & 30 Sep 88 & Thailand & 15 Sep 88 & 7 Jul 89 \\
\hline Malaysia & & 29 Aug 89 & & & \\
\hline
\end{tabular}

Asian States operating under Article 5 paragraph 1 of the Montreal Protocol: Bangladesh, China, Iran, Malaysia, Maldives, Philippines, Sri Lanka, Thailand.

\section{Amendment to the Montreal Protocol \\ London, 1990 \\ (Status as at 31 Jan. 1992, from UNEP)}

\begin{tabular}{lrr|llc} 
State & Sig. & Cons. & State & Sig. & Cons. \\
China & & 14 Jun 91 & Maldives & & 31 Jul 91 \\
Japan & & 4 Sep 91 & &
\end{tabular}

\section{HUMAN RIGHTS}

\section{International Convention on the Elimination of All Forms of Racial Discrimination \\ New York, 1966 \\ Entry into force: 4 Jan. 1969}

\begin{tabular}{lcc|lcr} 
State & Sig. & Cons. & State & Sig. & Cons. \\
$\begin{array}{l}\text { Afghanistan } \\
\text { Bangladesh }\end{array}$ & & 6 Jul 83 & Maldives & & 24 Apr 84 \\
Bhutan & & 11 Jun 79 & Mongolia & 3 May 66 & 6 Aug 69 \\
Cambodia & 26 Mar 73 & & Nepal & & 30 Jan 71 \\
China & 12 Apr 66 & 28 Nov 83 & Pakistan & 19 Sep 66 & 21 Sep 66 \\
India & & 29 Dec 81 & Papua New Guinea & & 27 Jan 82 \\
Iran & 2 Mar 67 & 3 Dec 68 & Philippines & 7 Mar 66 & 15 Sep 67 \\
Iraq & 8 Mar 67 & 29 Aug 68 & Seychelles & & 7 Mar 78 \\
Korea (Rep.) & 18 Feb 69 & 14 Jan 70 & Sri Lanka & & 18 Feb 82 \\
Laos. & 8 Aug 78 & 5 Dec 78 & Vietnam & & 9 Jun 82
\end{tabular}




\section{International Covenant on Economic, Social and Cultural Rights \\ New York, 1966 \\ Entry into force: 3 Jan. 1976}

\begin{tabular}{lll|lrr} 
State & Sig. & Cons. & State & Sig. & Cons. \\
Afghanistan & & 24 Jan 83 & Korea (Rep.) & & 10 Apr 90 \\
Cambodia & 17 Oct 80 & & Mongolia & 5 Jun 68 & 18 Nov 74 \\
India & & 10 Apr 79 & Philippines & 19 Dec 66 & 7 Jun 74 \\
Iran & 4 Apr 68 & 24 Jun 75 & Sri Lanka & & 11 Jun 80 \\
Japan & 30 May 78 & 21 Jun 79 & Vietnam & 24 Sep 82 \\
Korea (DPR) & & 14 Sep 81 & &
\end{tabular}

\section{International Covenant on Civil and Political Rights}

New York, 1966

Entry into force: 23 Mar. 1976

\begin{tabular}{lll|lrl} 
State & Sig. & Cons. & State & Sig. & Cons. \\
$\begin{array}{lccc}\text { Afghanistan } \\
\text { Cambodia }\end{array}$ & & 24 Jan 83 & Korea (Rep.) & & 10 Apr 90 \\
India & 17 Oct 80 & & Mongolia & 5 Jun 68 & 18 Nov 74 \\
Iran & & 10 Apr 79 & Philippines & 19 Dec 66 & 23 Oct 86 \\
Japan & 4 Apr 58 & 24 Jun 75 & Sri Lanka & & 11 Jun 80 \\
Korea (DPR) & 30 May 78 & 21 Jun 79 & Vietnam & & 24 Sep 82 \\
& & 14 Sep 81 & &
\end{tabular}

Optional Protocol to the International Covenant on Civil and Political Rights New York, 1966

Entry into force: 23 Mar. 1976

\begin{tabular}{lll|lll} 
State & Sig. & Cons. & State & Sig. & Cons. \\
Korea (Rep.) & & $10 \mathrm{Apr} 90$ & Philippines & $19 \mathrm{Dec} 66$ & 22 Aug 89
\end{tabular}

\section{Convention Against Torture and Other Cruel, Inhuman or Degrading Treatment or Punishment \\ New York, 1984}

Entry into force: 26 Jun. 1987

(Status as at 10 Dec. 1991, from E/CN.4/1992/15)

\begin{tabular}{|c|c|c|c|c|c|}
\hline State & Sig. & Cons. & State & Sig. & Cons. \\
\hline Afghanistan & 4 Feb 85 & 1 Apr 87 & Nepal & & 14 May 91 \\
\hline China & 12 Dec 86 & 4 Oct 88 & Philippines & & 18 Jun 86 \\
\hline Indonesia & 23 Oct 85 & & & & \\
\hline
\end{tabular}




\section{WOMEN AND CHILDREN}

\section{Convention on the Political Rights of Women \\ New York, 1953 \\ Entry into force: 7 Jul. 1954}

\begin{tabular}{lcc|lcc} 
State & Sig. & Cons. & State & Sig. & Cons. \\
Afghanistan & & 16 Nov 66 & Myanmar & 14 Sep 54 & \\
India & 29 Apr 53 & 1 Nov 61 & Nepal & & 25 Apr 65 \\
Indonesia & 31 Mar 53 & 16 Dec 58 & Pakistan & 18 May 54 & 7 Dec 54 \\
Japan & 1 Apr 55 & 13 Jul 55 & Papua New Guinea & & 27 Jan 82 \\
Korea (Rep.) & & 23 Jun 59 & Philippines & 23 Sep 53 & 12 Sep 57 \\
Laos & & 28 Jan 69 & Thailand & 5 Mar 54 & 30 Nov 54 \\
Mongolia & & 18 Aug 65 & & &
\end{tabular}

\section{Convention on the Nationality of Married Women}

New York, 1957

Entry into force: 11 Aug. 1958

\begin{tabular}{|c|c|c|c|c|c|}
\hline State & Sig. & Cons. & State & Sig. & Cons. \\
\hline India & 15 May 57 & & Singapore & & $18 \operatorname{Mar} 66$ \\
\hline Malaysia & & $24 \mathrm{Feb} 59$ & Sri Lanka & & 30 May 58 \\
\hline Pakistan & $10 \mathrm{Apr} 58$ & & & & \\
\hline
\end{tabular}

\section{Convention on the Elimination of All Forms of Discrimination Against Women New York, 1980}

Entry into force: 3 Sep. 1981

\begin{tabular}{|c|c|c|c|c|c|}
\hline State & Sig. & Cons. & State & Sig. & Cons. \\
\hline Afghanistan & 14 Aug 80 & & Korea (Rep.) & 25 May 83 & 27 Dec 84 \\
\hline Bangladesh & & 6 Nov 84 & Laos & 17 Jul 80 & 14 Aug 81 \\
\hline Bhutan & 17 Jul 80 & 31 Aug 81 & Mongolia & $17 \mathrm{Jul} 80$ & 20 JuI 81 \\
\hline Cambodia & 17 Oct 80 & & Philippines & $15 \mathrm{Jul} 80$ & 5 Aug 81 \\
\hline China & $17 \mathrm{Jul} 80$ & 4 Nov 80 & Sri Lanka & $17 \mathrm{Jul} 80$ & 5 Oct 81 \\
\hline India & 30 Jul 80 & & Thailand & & 9 Aug 85 \\
\hline Indonesia & 29 Jul 80 & 13 Sep 84 & Vietnam & 29 Jul 80 & 17 Feb 82 \\
\hline Japan & $17 \mathrm{Jul} 80$ & 25 Jun 85 & & & \\
\hline
\end{tabular}




\section{Convention on the Rights of the Child \\ New York, 1989 \\ Entry into force: 2 Sep. 1990}

(Status as at 16 Dec. 1991, from E/CN.4/1992/54)

\begin{tabular}{|c|c|c|c|c|c|}
\hline State & Sig. & Cons. & State & Sig. & Cons. \\
\hline Afghanistan & $27 \operatorname{Sep} 90$ & & Mongolia & $26 \operatorname{Jan} 90$ & 5 Jul 90 \\
\hline Bangladesh & $26 \operatorname{Jan} 90$ & 3 Aug 90 & Myanmar & & 15 Jul 91 \\
\hline Bhutan & 4 Jun 90 & 1 Aug 90 & Nepal & $26 \operatorname{Jan} 90$ & $14 \operatorname{Sep} 90$ \\
\hline China & 29 Aug 90 & & Pakistan & $20 \operatorname{Sep} 90$ & 12 Nov 90 \\
\hline Indonesia & $25 \operatorname{Jan} 90$ & $5 \operatorname{Sep} 90$ & Papua New Guinea & 30 Sep 90 & \\
\hline Japan & 21 Sep 90 & & Philippines & $26 \mathrm{Jan} 90$ & 21 Aug 90 \\
\hline Korea (DPR) & 23 Aug 90 & $21 \operatorname{Sep} 90$ & Seychelles & & 7 Sep 90 \\
\hline Korea (Rep.) & $25 \operatorname{Sep} 90$ & 20 Nov 91 & Sri Lanka & $26 \operatorname{Jan} 90$ & $12 \mathrm{Jul} 91$ \\
\hline Laos & & 8 May 91 & Vietnam & $26 \operatorname{Jan} 90$ & $28 \mathrm{Feb} 90$ \\
\hline Maldives & 21 Aug 90 & $11 \mathrm{Feb} 91$ & & & \\
\hline
\end{tabular}

\section{REFUGEES}

\section{Convention Relating to the Status of Refugees}

Geneva, 1951

Entry into force: 22 Apr. 1954

\begin{tabular}{|c|c|c|c|c|c|}
\hline State & Sig. & Cons. & State & Sig. & Cons. \\
\hline China & & $24 \operatorname{Sep} 82$ & Papua New Guinea & & 17 Jul 86 \\
\hline Iran & & 28 Jul 76 & Philippines & & $22 \mathrm{Jul} 81$ \\
\hline Japan & & 3 Oct 81 & Seychelles & & $23 \mathrm{Apr} 8 \mathrm{C}$ \\
\hline
\end{tabular}

\section{Protocol Relating to the Status of Refugees}

New York, 1967

Entry into force: 4 Oct. 1967

\begin{tabular}{lcc|lll} 
State & Sig. & Cons. & State & Sig. & Cons. \\
China & & 24 Sep 82 & Papua New Guinea & & 17 Jul 86 \\
Iran & & 28 Jul 76 & Philippines & & 22 Jul 81 \\
Japan & 1 Jan 82 & Seychelles & & 23 Apr 80
\end{tabular}




\section{HUMANITARIAN LAW IN ARMED CONFLICT}

\section{International Conventions for the Protection of Victims of War, I-IV}

Geneva, 1949

Entry into force: 21 Oct. 1950

(Dates refer to the day on which the official deed was received by the Swiss Federal Department of Foreign Affairs.

Data as at 30 June 1990 from DDM/JUR 90/823 - CPS/4 (Comité International de la Croix-Rouge); data as at 21 June 1991 courtesy of Netherlands Red Cross Society)

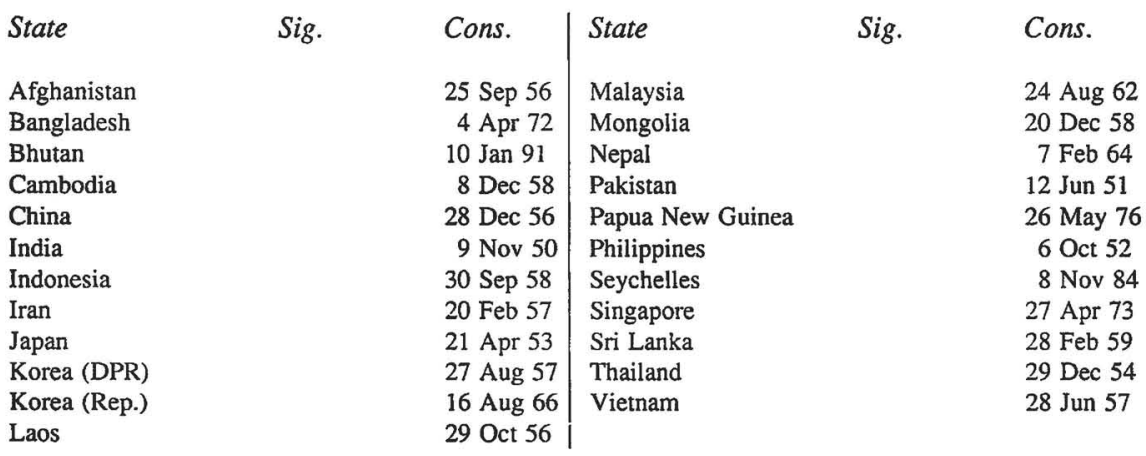

\section{Protocol I Additional to the Geneva Conventions of 12 August 1949, and Relating to the Protection of Victims of International Armed Conflicts Geneva, 1977}

Entry into force: 7 Dec. 1978

(Dates refer to the day on which the official deed was received by the Swiss Federal Department of Foreign Affairs.

Data as at 30 June 1990 from DDM/JUR 90/823 - CPS/4 (Comité

International de la Croix-Rouge); data as at 21 June 1991 courtesy of Netherlands Red Cross Society; data as at 17 July 1991 from A/INF/46/3)

\begin{tabular}{|c|c|c|c|c|c|}
\hline State & Sig. & Cons. & State & Sig. & Cons. \\
\hline Bangladesh & & 8 Sep 80 & Laos & & 18 Nov 80 \\
\hline China & & $14 \operatorname{Sep} 83$ & Seychelles & & 8 Nov 84 \\
\hline Korea (DPR) & & 9 Mar 88 & Vietnam & & 19 Nov 81 \\
\hline Korea (Rep.) & & 15 Jan 82 & & & \\
\hline
\end{tabular}


Protocol II Additional to the Geneva Conventions of 12 August 1949, and Relating to the Protection of Victims of Non-International Armed Conflicts Geneva, 1977

Entry into force: 7 Dec. 1978

(Dates refer to the day on which the official deed was received by the Swiss Federal Department of Foreign Affairs.

Data as at 30 June 1990 from DDM/JUR 90/823 - CPS/4 (Comité International de la Croix-Rouge); data as at 21 June 1991 courtesy of Netherlands Red Cross Society; data as at 17 July 1991 from A/INF/46/3)

\begin{tabular}{lrr|lrr} 
State & Sig. & Cons. & State & Sig. & Cons. \\
Bangladesh & & & 18 Nov 80 \\
China & & 8 Sep 80 & Laos & & 11 Dec 86 \\
Korea (Rep.) & & 14 Sep 83 & Philippines & & 8 Nov 84
\end{tabular}

\section{INTERNATIONAL CRIMES}

\section{Convention on the Prevention and Punishment of the Crime of Genocide}

New York, 1948

Entry into force: 12 Jan. 1951

\begin{tabular}{lll|llc} 
State & Sig. & Cons. & State & Sig. & Cons. \\
Afghanistan & & 22 Mar 56 & Mongolia & & 5 Jan 67 \\
Cambodia & & 14 Oct 50 & Myanmar & 30 Dec 49 & 14 Mar 56 \\
China & 20 Jul 49 & 18 Apr 83 & Nepal & & 17 Jan 69 \\
India & 29 Nov 49 & 27 Aug 59 & Pakistan & 11 Dec 48 & 12 Oct 57 \\
Iran & 8 Dec 49 & 14 Aug 56 & Papua New Guinea & & 27 Jan 82 \\
Korea (DPR) & & 31 Jan 89 & Philippines & 11 Dec 48 & 7 Jul 50 \\
Korea (Rep.) & & 14 Oct 50 & Sri Lanka & & 12 Oct 50 \\
Laos & & 8 Dec 50 & Vietnam & & 9 Jun 81 \\
Maldives & & 24 Apr 84 & &
\end{tabular}

\section{Convention on Offences and Certain Other Acts Committed on Board Aircraft} Tokyo, 1963

Entry into force: 4 Dec. 1969

(Information furnished on 16 July 1991 by the Secretariat of ICAO and reproduced in $\mathrm{A} / 46 / 346$ )

\begin{tabular}{lll|l} 
State & Cons. & $\begin{array}{l}\text { Effective } \\
\text { date }\end{array}$ & State \\
Afghanistan & 15 Apr 77 & 14 Jul 77 & China \\
Bangladesh & 25 Jul 78 & 23 Oct 78 & India \\
Bhutan & $25 \mathrm{Jan} 89$ & 25 Apr 89 & Indonesia \\
Brunei & 23 May 86 & 21 Aug 86 &
\end{tabular}

$\begin{array}{ll}\text { Cons. } & \begin{array}{l}\text { Effective } \\ \text { date }\end{array} \\ & \\ \text { 14 Nov } 78 & 12 \text { Feb } 79 \\ \text { 22 Jul } 75 & 20 \text { Oct } 75 \\ 7 \text { Sep } 76 & 6 \text { Dec } 76 \\ \text { (sig. 14 Sep 63) }\end{array}$




\begin{tabular}{|c|c|c|c|c|c|}
\hline State & Cons. & $\begin{array}{l}\text { Effective } \\
\text { date }\end{array}$ & State & Cons. & $\begin{array}{l}\text { Effective } \\
\text { date }\end{array}$ \\
\hline Iran & 28 Jun 76 & $29 \operatorname{Sep} 76$ & Pakistan & 11 Sep 73 & $10 \operatorname{Dec} 73$ \\
\hline \multirow[t]{2}{*}{ Japan } & 26 May 70 & 24 Aug 70 & & (sig. 6 Aug 6 & \\
\hline & \multicolumn{2}{|c|}{ (sig. 14 Sep 63) } & Papua New Guinea & $-?-$ & $16 \operatorname{Sep} 75$ \\
\hline Korea (DPR) & 9 May 83 & 7 Aug 83 & Philippines & 26 Nov 65 & $4 \operatorname{Dec} 69$ \\
\hline \multirow[t]{2}{*}{ Korea (Rep.) } & 19 Feb 71 & 20 May 71 & & (sig. $14 \mathrm{Sep}$ & \\
\hline & \multicolumn{2}{|c|}{ (sig. $8 \mathrm{Dec} 65$ ) } & Seychelles & $4 \operatorname{Jan} 79^{\circ}$ & 4 Apr 79 \\
\hline Laos & 23 Oct 72 & $21 \mathrm{Jan} 73$ & Singapore & 1 Mar 71 & 30 May 71 \\
\hline Malaysia & 5 Mar 85 & 3 Jun 85 & Sri Lanka & 30 May 78 & 28 Aug 78 \\
\hline Maldives & $28 \operatorname{Sep} 87$ & $21 \operatorname{Dec} 87$ & Thailand & $6 \operatorname{Mar} 72$ & $4 \operatorname{Jun} 72$ \\
\hline Mongolia & 24 Jul 90 & 22 Oct 90 & Vietnam & 10 Oct 79 & $8 \mathrm{Jan} 80$ \\
\hline pal & 15 Jan 79 & 15 Apr 79 & & & \\
\hline
\end{tabular}

\section{Convention for the Suppression of Unlawful Seizure of Aircraft}

The Hague, 1970

Entry into force: 14 Oct. 1971

(Information furnished on 16 July 1991 by the Secretariat of ICAO and reproduced in $\mathrm{A} / 46 / 346$ )

\begin{tabular}{|c|c|c|c|c|c|}
\hline State & Sig. & Cons. & State & Sig. & Cons. \\
\hline Afghanistan & $16 \operatorname{Dec} 70$ & 29 Aug 79 & Malaysia & $16 \operatorname{Dec} 70$ & 4 May 85 \\
\hline Bangladesh & & 28 Jun 78 & Maldives & & 1 Sep 87 \\
\hline Bhutan & & 28 Dec 88 & Mongolia & $18 \operatorname{Jan} 71$ & 8 Oct 71 \\
\hline Brunei & & 16 Apr 86 & Nepal & & 11 Jan 79 \\
\hline Cambodia & $16 \operatorname{Dec} 70$ & & Pakistan & 12 Aug 71 & 28 Nov 73 \\
\hline China & & $10 \mathrm{Sep} 80$ & Papua New Guinea & & $15 \operatorname{Dec} 75$ \\
\hline India & $14 \mathrm{Jul} 71$ & 12 Nov 82 & Philippines & $16 \operatorname{Dec} 70$ & $26 \mathrm{Mar} 73$ \\
\hline Indonesia & $16 \operatorname{Dec} 70$ & 27 Aug 76 & Seychelles & & $29 \operatorname{Dec} 78$ \\
\hline Iran & $16 \operatorname{Dec} 70$ & $25 \mathrm{Jan} 72$ & Singapore & $8 \operatorname{Sep} 71$ & 12 Apr 78 \\
\hline Japan & 16 Dec 70 & 19 Apr 71 & Sri Lanka & & 30 May 78 \\
\hline Korea (DPR) & & $28 \mathrm{Apr} 83$ & Thailand & $16 \operatorname{Dec} 70$ & 16 May 78 \\
\hline Korea (Rep.) & & $18 \mathrm{Jan} 73$ & Vietnam & & $17 \operatorname{Sep} 79$ \\
\hline Laos & 16 Feb 71 & 6 Apr 89 & & & \\
\hline
\end{tabular}

\section{Convention for the Suppression of Unlawful Acts Against the Safety of Civil Aviation \\ Montreal, 1971}

Entry into force: 26 Jan 1973

(Information furnished on 16 July 1991 by the Secretariat of the ICAO and reproduced in A/46/346)

State
Afghanistan
Bangladesh
Bhutan
Brunei
China

\begin{tabular}{ll|l} 
Sig. & Cons. & State \\
& 26 Sep 84 & India \\
28 Jun 78 & Indonesia \\
28 Dec 88 & Iran \\
16 Apr 86 & Japan \\
10 Sep 80 & Korea (DPR)
\end{tabular}

Sig. Cons. 


\begin{tabular}{lrr|lll} 
State & Sig. & Cons. & State & Sig. & Cons. \\
Korea (Rep.) & & 2 Aug 73 & Philippines & 23 Sep 71 & 26 Mar 73 \\
Laos & 1 Nov 72 & 6 Apr 89 & Seychelles & & 29 Dec 78 \\
Maldives & & 1 Sep 87 & Singapore & 21 Nov 72 & 12 Apr 78 \\
Mongolia & 19 Feb 72 & 14 Sep 72 & Sri Lanka & & 30 May 78 \\
Nepal & & 11 Jan 79 & Thailand & 16 May 78 \\
Pakistan & & 24 Jan 74 & Vietnam & 17 Sep 79 \\
Papua New Guinea & & 15 Dec 75 & &
\end{tabular}

\section{International Convention Against the Taking of Hostages New York, 1979 \\ Entry into force: 3 Jun. 1983}

\begin{tabular}{llc|llr} 
State & Sig. & Cons. & State & Sig. & Cons. \\
Bhutan & & 31 Aug 81 & Korea (Rep.) & & 4 May 83 \\
Brunei & & 18 Oct 88 & Philippines & 2 May 80 & 14 Oct 80 \\
Japan & 22 Dec 80 & 8 Jun 87 & & &
\end{tabular}

\section{Convention for the Suppression of Unlawful Acts Against the Safety of Maritime Navigation \\ Rome, 1988 \\ (Information furnished on 16 July 1991 by the Secretariat of IMO} and reproduced in $\mathrm{A} / 46 / 346$ )

\begin{tabular}{lll|lll} 
State & Sig. & Cons. & State & Sig. & Cons. \\
Brunei & 3 Feb 88 & & Philippines & 10 Mar 88 & \\
China & 25 Oct 88 & & Seychelles & 24 Jan 89 & 24 Jan 89
\end{tabular}

\section{Protocol for the Suppression of Unlawful Acts Against the Safety of Fixed Platforms Located on the Continental Shelf Rome, 1988 \\ (Information furnished on 16 July 1991 by the Secretariat of IMO and reproduced in $\mathrm{A} / 46 / 346$ )}

\begin{tabular}{lll|lll} 
State & Sig. & Cons. & State & Sig. & Cons. \\
Brunei & 3 Feb 89 & & Philippines & 10 Mar 88 & \\
China & 25 Oct 88 & & Seychelles & 24 Jan 89 & 24 Jan 89
\end{tabular}




\section{Protocol for the Suppression of Unlawful Acts of Violence at Airports Serving International Civil Aviation, Supplementary to the Convention for the Suppression of Unlawful Acts Against the Safety of Civil Aviation \\ Montreal, 1988 \\ Entry into force: 6 Aug. 1989 \\ (Information is reproduced below as furnished on 16 July 1991 by the Secretariat of the ICAO and reproduced in $\mathrm{A} / 46 / 346$ )}

\begin{tabular}{lll|lll} 
State & Sig. & Cons. & State & Sig. & Cons. \\
China & 24 Feb 88 & & Malaysia & 24 Feb 88 & \\
Indonesia & 24 Feb 88 & & Pakistan & 24 Feb 88 & \\
Korea (DPR) & 11 Apr 89 & Philippines & 25 Jan 89 & \\
Korea (Rep.) & 24 Feb 88 & 27 Jun 90 & Sri Lanka & 28 Oct 88 &
\end{tabular}

Convention on the Marking of Plastic Explosives for the Purpose of Detection Montreal, 1991

(Information furnished on 16 July 1991 by the Secretariat of ICAO and reproduced in $\mathrm{A} / 46 / 346$ )

\begin{tabular}{lll|lll} 
State & Sig. & Cons. & State & Sig. & Cons. \\
Pakistan & $1 \mathrm{Mar} 91$ & & Korea (Rep.) & $1 \mathrm{Mar} 91$ &
\end{tabular}

\section{NARCOTIC DRUGS}

Convention for the Suppression of the Illicit Traffic in Dangerous Drugs Geneva, 1936, amended by Protocol, Lake Success, New York, 1946. Entry into force: 10 Oct. 1947

\begin{tabular}{llc|lcc} 
State & Sig. & Cons. & State & Sig. & Cons. \\
Cambodia & & 3 Oct 51 & Japan & & 7 Sep 55 \\
China & 11 Dec 46 & & Laos & & 13 Jul 51 \\
India & 11 Dec 46 & & Sri Lanka & & 4 Dec 57 \\
Indonesia & & 3 Apr 58 & &
\end{tabular}




\section{Single Convention on Narcotic Drugs \\ New York, 1961 \\ Entry into force: 13 Dec. 1964}

\begin{tabular}{llc|lcc} 
State & Sig. & Cons. & State & Sig. & Cons. \\
$\begin{array}{l}\text { Afghanistan } \\
\text { Bangladesh }\end{array}$ & 30 Mar 61 & 19 Mar 63 & Malaysia & & 11 Jul 67 \\
Brunei & & 25 Apr 75 & Myanmar & 30 Mar 61 & 29 Jul 63 \\
Cambodia & 25 Nov 87 & Nepal & & 29 Sep 87 \\
India & 30 Mar 61 & & Pakistan & 30 Mar 61 & 9 Jul 65 \\
Indonesia & 30 Mar 61 & 13 Dec 64 & Papua New Guinea & & 28 Oct 80 \\
Iran & 28 Jul 61 & 3 Sep 76 & Philippines & 30 Mar 61 & 2 Oct 67 \\
Japan & 30 Mar 61 & 30 Aug 72 & Singapore & & 15 Mar 73 \\
Korea (Rep.) & 26 Jul 61 & 13 Jul 64 & Sri Lanka & & 11 Jul 63 \\
Laos & 30 Mar 61 & 13 Feb 62 & Thailand & 24 Jul 61 & 31 Oct 61
\end{tabular}

Single Convention on Narcotic Drugs, 1961, as Amended by the Protocol of 25 March 1972 Amending the Single Convention on Narcotic Drugs, 1961 New York, 1975

Entry into force: 8 Aug. 1975

\begin{tabular}{lrl|lll} 
State & Sig. & Cons. & State & Sig. & Cons. \\
Bangladesh & 9 May 80 & & Philippines & 7 Jun 74 \\
$\begin{array}{l}\text { Brunei } \\
\text { Malaysia }\end{array}$ & 25 Nov 87 & & Singapore & 9 Jul 75 \\
$\begin{array}{l}\text { Nepal } \\
\text { Papua New Guinea }\end{array}$ & 20 Apr 78 & & Sri Lanka & 29 Jun 81 \\
\end{tabular}

\section{United Nations Convention Against Illicit Traffic in Narcotic Drugs and Psychotropic Substances}

Vienna, 1988

Entry into force: 11 Nov. 1990

\begin{tabular}{|c|c|c|c|c|c|}
\hline State & Sig. & Cons. & State & Sig. & Cons. \\
\hline Afghanistan & 20 Dec 88 & & Iran & $20 \operatorname{Dec} 88$ & \\
\hline Bangladesh & 14 Apr 89 & 11 Oct 90 & Japan & 19 Dec 89 & \\
\hline Bhutan & & 27 Aug 90 & Malaysia & $20 \mathrm{Dec} 88$ & \\
\hline Brunei & 26 Oct 89 & & Maldives & $5 \operatorname{Dec} 89$ & \\
\hline China & 20 Dec 88 & 25 Oct 89 & Myanmar & & 11 Jun 91 \\
\hline India & & $27 \operatorname{Mar} 90$ & Pakistan & 20 Dec 89 & \\
\hline Indonesia & 27 Mar 89 & & Philippines & $20 \operatorname{Dec} 88$ & \\
\hline
\end{tabular}




\section{NUCLEAR MATERIAL}

\section{Convention on the Physical Protection of Nuclear Material} Vienna, 1980

Entry into force: 8 Feb. 1987

(Information furnished on 17 July 1991 by the Secretariat of IAEA and reproduced in $\mathrm{A} / 46 / 346)$

\begin{tabular}{llc|llc} 
State & Sig. & Cons. & State & Sig. & Cons. \\
China & & 10 Jan 89 & Korea (Rep.) & 29 Dec 81 & 7 Apr 82 \\
Indonesia & 3 Jul 86 & 5 Nov 86 & Mongolia & 23 Jan 86 & 28 May 86 \\
Japan & & 28 Oct 88 & Philippines & 19 May 80 & 22 Sep 81
\end{tabular}

